\title{
Uso de las revistas-e suministradas por Emerald en bibliotecas universitarias españolas (2002-2005)
}

\author{
Por Blanca Rodríguez Bravo y Ma Luisa Alvite Díez
}

\begin{abstract}
Resumen: Estudio del uso de las revistas electrónicas distribuidas por Emerald en las universidades de Burgos, La Coruña, La Rioja, León, Oviedo, Valladolid y Vigo en los primeros años de su contratación, 2002-2005. El análisis se vale de las estadísticas que el distribuidor proporciona a los bibliotecarios y se circunscribe al estudio de los datos de artículos descargados. Las conclusiones esbozadas en el presente trabajo se refieren fundamentalmente a los patrones de uso de universidades de distinto tamaño y a la calidad de los contenidos distribuidos por Emerald: dispersión de revistas utilizadas, núcleo de publicaciones y relevancia de éstas últimas.
\end{abstract}

Palabras clave: Bibliotecas universitarias, Revistas electrónicas, Uso de revistas electrónicas, Emerald, España.

Title: Usage in Spanish academic libraries of electronic journals provided by Emerald (2002-2005)

Abstract: Study of the usage of electronic journals distributed by Emerald to universities of Burgos, La Coruña, LaRioja, León, Oviedo, Valladolid and Vigo during the initial years of subscription, 2002-2005. The analysis uses statistics provided by the distributor and is restricted to the study of data concerning downloaded articles. The conclusions outlined in the present work refer fundamentally to the patterns of use within universities of different sizes and to the quality of the contents distributed by Emerald: spread of journals used, core publications, and journal relevance.

Keywords: Academic libraries, Electronic journals, Usage of electronic journals, Emerald, Spain.

Rodríguez Bravo, Blanca; Alvite Díez, Ma Luisa. "El uso de las revistas-e suministradas por Emerald en bibliotecas universitarias españolas (2002-2005)". En: El profesional de la información, 2006 noviembre-diciembre, v. 15, n. 6, pp. 464-472.

\section{Introducción}

ESTE TRABAJO SE ENMARCA EN UN CORPUS de bibliografía sobre estudios de uso y de usuarios de recursos electrónicos, en la actualidad bastante abundante, y que ha sido revisado y consolidado por Tenopir (2003) y por los documentos recogidos en las actas del seminario que el Ingenta Institute (2002) dedicó justamente al tema de las compras consorciadas y a su rendimiento.

Los paquetes de revistas se contrataron, generalmente, siguiendo el modelo que se ha dado en llamar Big Deal, dirigido especialmente a consorcios a los que se ofrece incrementar espectacularmente la accesibilidad a la información científica, rompiendo la tendencia anterior de recortes continuos en las colecciones bibliotecarias de publicaciones periódicas. La adquisición de revistas siguiendo este patrón ha reportado, además de beneficios económicos, un incremento real en la dispersión de uso de los fondos disponibles. Dicho modelo ha sido objeto de diversos estudios que nos han proporcionado un marco de referencia (Frazier, 2001a, 2001b; Rowse, 2003; Sanville, 2001; Urbano et al., 2004).

Aunque se carece de datos del número global de títulos que constituía la colección de revistas de las bibliotecas españolas en los inicios del milenio, puede afirmarse a tenor de los estudios realizados (Urbano et al., 2004) la amplitud de la ganancia proporcionada por el modelo Big Deal, dado que la presión inflacionista de las publicaciones periódicas estaba conduciendo a las bibliotecas a continuas cancelaciones.

Además del gran incremento de la accesibilidad a revistas científicas se constata la flexibilidad de uso de la información, dado que resulta notoria la utilización de títulos que no se poseían en papel, en muchos casos priorizados sobre aquellos, como se ha podido apreciar en los datos de que disponemos correspondientes a las universidades de Burgos y León (Rodríguez; Alvite, 2005b). Este aspecto parece haberse confirmado, asimismo, en las universidades de Ohio, donde, como señala Sanville (2001), 2/3 de los títulos usados no se encontraban disponibles previamente en papel.

El mismo autor resalta las ventajas del crecimiento en el número de publicaciones periódicas disponibles y señala que asistimos en nuestras bibliotecas a un importante cambio de mentalidad de "sé lo que mis usuarios necesitan" a "vamos a ver qué necesitan mis usuarios". La nueva visión requiere una más amplia disponibilidad y accesibilidad de documentos que promuevan un mayor uso.

Es en este contexto donde se ha de situar la explotación de estadísti- 
cas de recursos electrónicos, así como la evaluación del rendimiento de la inversión y de la satisfacción de los usuarios. La utilidad de los datos obtenidos a partir del trabajo de recogida, explotación y análisis de las estadísticas detalladas y exhaustivas del uso de las revistas ha sido sintetizada por Peters (2002) en razones de carácter académico, causas de planificación y evaluación de servicios bibliotecarios, razones de tipo económico en el ámbito de la gestión interna de los consorcios y de las bibliotecas participantes y, por último, motivaciones de carácter económico en el ámbito de la renegociación de licencias.

Las conclusiones esbozadas en el presente trabajo se refieren fundamentalmente a los patrones de uso de universidades de distinto tamaño y a la calidad de los contenidos distribuidos por Emerald: dispersión de revistas utilizadas, núcleo de publicaciones y relevancia de éstas últimas. Asimismo, se podrán extraer conclusiones sobre la necesidad de incidir en la tarea de transmisión de los recursos existentes por parte de la biblioteca que permita potenciar el uso de los contenidos electrónicos. Como afirma Sanville (2001) en el primer estadio de la implantación de la biblioteca digital los usuarios no han asimilado las posibilidades informativas que los paquetes electrónicos les proporcionan. Este mismo autor señala que añadiendo títulos a los paquetes, enlaces bibliográficos $\mathrm{y}$ mejorando las opciones de búsqueda se incrementará la descarga de artículos.

El examen de la cobertura temática de los contenidos, así como los aspectos técnicos y funcionales de los principales distribuidores de contenidos electrónicos ha sido llevado a cabo previamente por las autoras (Rodríguez; Alvite, 2004, 2005; Alvite; Rodríguez, 2005) pues, como confirman Eason, Ri- chardson y Yu (2000, pp. 477, 499) el uso resulta condicionado por la influencia de los contenidos (cobertura y relevancia) y la facilidad de utilización.

\section{Objetivos y metodología}

El objetivo general se dirige a estudiar el consumo de la información electrónica de las comunidades universitarias de Burgos, La Coruña, La Rioja, León, Oviedo, Valladolid y Vigo que podría redundar en beneficio del fortalecimiento de la negociación de las bibliotecas universitarias con los proveedores de recursos electrónicos y en la toma fundamentada de decisiones en la contratación e inversión de presupuestos limitados. El trabajo, en concreto, permitirá conocer:

-Qué se está usando: datos sobre el número de artículos descargados, desglosados por títulos que permitirán a los bibliotecarios valorar el interés de la suscripción mantenida.

—Quién usa la información: el análisis de los títulos más usados permite obtener datos relevantes a la hora de determinar las necesidades de información de las distintas comunidades universitarias, las necesidades de formación de los usuarios o la conveniencia de dar más visibilidad al producto.

Los objetivos específicos perseguidos son los siguientes:

- Determinar el uso de la colección de revistas electrónicas de Emerald en varias universidades españolas.

- Valorar las diferencias de uso que de los contenidos electrónicos realizan las distintas universidades.

- Analizar la concentración y/o dispersión del uso de los contenidos electrónicos contratados inducido por un consumo más libre, exploratorio y accidental de las colecciones.
- Contrastar la calidad y relevancia de las publicaciones más utilizadas, atendiendo a los parámetros fijados por el Institut for Scientific Information (ISI).

Se analiza el uso de las revistas electrónicas distribuidas por Emerald, una empresa cuyo origen se remonta a 1967, año en el que se crea MCB University Press. La editorial, desde sus inicios, centró su interés en disciplinas relacionadas con la gestión y el marketing. En 2001 adopta el nombre de Emerald, empleando el de su producto estrella: The electronic management research library database.

En el momento actual la empresa señala la distribución, con carácter dinámico, de unas 150 revistas electrónicas; se trata de un paquete dirigido mayoritariamente a la distribución de contenidos adscribibles a las ciencias sociales y, primordialmente, a las áreas de economía y ciencias de la información. No obstante, se oferta un número que podemos considerar limitado de títulos pertenecientes al campo de las ciencias técnicas.

El análisis de la interfaz de este proveedor abordado en un trabajo previo (Alvite; Rodríguez, 2005) subrayaba aspectos positivos relacionados con la recuperación conceptual, disponiendo para tal fin de herramientas terminológicas auxiliares, haciendo uso de materias, clasificación e índice inverso de palabras clave, etc.

Como se ha señalado, las universidades contempladas en el estudio son las de Burgos, La Coruña, La Rioja, León, Oviedo y Vigo. En el caso de la de Valladolid sólo disponemos de datos de descargas globales que hemos considerado adecuado incluir por permitir una comparación propicia con los obtenidos de la de Oviedo, por tratarse de instituciones de dimensiones similares.

Los tamaños de las universidades analizadas son dispares, siendo 


\begin{tabular}{|c|c|c|c|c|}
\hline Universidad & Alumnos & $\begin{array}{c}\text { *Personal } \\
\text { docente- } \\
\text { investigador }\end{array}$ & $\begin{array}{c}\text { Titulaciones } \\
\text { oficiales }\end{array}$ & $\begin{array}{c}\text { Titulaciones en } \\
\text { ciencias sociales }\end{array}$ \\
\hline Burgos & 9.177 & 544 & 31 & 17 \\
\hline La Coruña & 24.012 & 1.215 & 49 & 20 \\
\hline La Rioja & 6.889 & 371 & 26 & 11 \\
\hline León & 14.205 & 833 & 53 & 23 \\
\hline Oviedo & 31.615 & 1.965 & 69 & 49 \\
\hline Valladolid & 31.780 & 2.024 & 99 & 26 \\
\hline Vigo & 26.143 & 1.341 & 56 & \\
\hline
\end{tabular}

Tabla 1. Datos de universidades curso 2005/2006

las cifras de personal docente-investigador proporcionales a las del alumnado en todas las instituciones $^{1}$ (tabla 1).

Todas las instituciones objeto de estudio disponen de un núcleo considerable de titulaciones de ciencias sociales, 11 en La Rioja, 17 en Burgos y superior a la veintena en las universidades restantes. La Rioja es la institución más pequeña y no alcanza los 7.000 alumnos en los cursos 2004-2005 y 2005-2006. Le siguen los centros de Burgos, con un alumnado en torno a los 9.000 estudiantes y el de León con aproximadamente 14.000 . De tamaño medio son las dos universidades gallegas consideradas, La Coruña y Vigo, con cerca de los 25.000 estudiantes la primera y por encima de esta cifra la segunda. Las universidades de Oviedo y Valladolid superan los 30.000, 31.000 en el curso 2005-2006, si bien en 2004-2005 Valladolid no alcanzaba la cantidad de 30.000 que era superada por Oviedo.

\section{Recogida de datos}

Se contabilizan los datos de uso de las revistas de Emerald a partir de dos fuentes de datos facilitadas por el editor: por un lado, ficheros cronológicos de uso global de todas las publicaciones disponibles en el portal y, por otro, ficheros detallados por títulos, a partir de los cuales se podrá calcular exactamente el uso imputable al paquete contratado. Los títulos usados tienen en cuenta exclusivamente las descargas efectuadas, no los posibles accesos: artículos visualizados, abstracts, etc.

Cabe reseñar la disparidad en la cifra de revistas contratadas por las distintas universidades, que oscilan entre las 76 de La Coruña y las 145 de Oviedo.

El período cronológico de recogida de datos comprende desde 2002 hasta 2005. Son las universidades castellano-leonesas las que cuentan con una más larga trayectoria en la disposición de este suministrador. Por el contrario, la contratación en La Coruña comienza en 2005. Los datos de descargas por títulos se han ceñido al año 2005 por considerarse alcanzado un grado suficiente de consolidación en el uso de las publicaciones electrónicas en el conjunto de las universidades.

Una vez revisados los principales estándares para la recogida y la construcción de indicadores de uso de información y servicios electrónicos -Counter, E-metrics, Equinox, Icolc, ISO 2789, ISO 20983, NISO Z39.7- decidimos formalizar los indicadores de rendimiento estructurados en dos niveles:

-Datos de descargas globales de artículos desglosados por institución/año.
-Datos de descargas de cada título de revista desglosados por institución en 2005.

\section{Tratamiento y análisis de datos}

Para estudiar el corpus de datos resultante se ha dirigido el esfuerzo a los puntos siguientes:

a. Datos de descargas globales de artículos desglosados por institución/año.

a.1. Artículos descargados por institución anualmente.

b. Datos de descargas de cada título de revista desglosados por institución en 2005.

b.1. Títulos suscritos por institución.

b.2. Títulos usados por institución.

b.3. Núcleo de títulos por institución.

b.4. Dispersión y concentración de uso.

c. Análisis de los títulos más empleados en el conjunto de las instituciones en 2005.

c.1. Núcleo de títulos en el conjunto de las universidades estudiadas.

c.2. Relevancia de las publicaciones atendiendo al factor de impacto establecido por el ISI. 


\section{doc6 en 30 segundos}

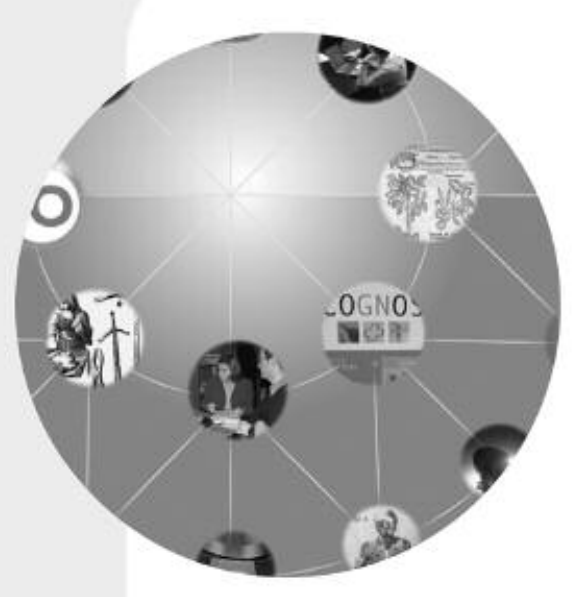

\section{La empresa}

Creada en el año 1988, especializada en gestión de la información y la documentación.

Certificada con la ISO 9001:2000 por Bureau Veritas desde Septiembre del 2000 en las siguientes actividades:

CONSULTORES EN RECURSOS DE INFORMACIÓN
GESTIÓN DEL CONOCIMIENTO
INTRANET/EXTRANET/INTERNET
EDICIÓN ELECTRÓNICA

PROVEEDORES DE RECURSOS ELECTRÓNICOS DE INFORMACIÓN

SOLUCIONES EN GESTIÓN DOCUMENTAL

- CURSOS DE FORMACIÓN

\section{Nuestros valores}

Innovación permanente.

Satisfacción del cliente. Calidad

Profesionalidad

Cooperación con el cliente

Confidencialidad

Responsabilidad social y valores éticos

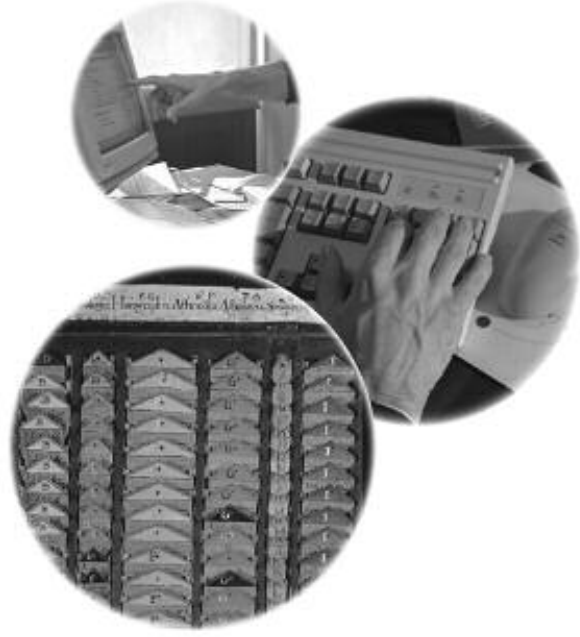

\section{Los clientes}

El principal activo de la empresa, junto con sus empleados.

Más de 900 clientes: empresas, laboratorios, administraciones públicas, universidades, hospitales, bufetes de abogados, museos, fundaciones, colegios profesionales.

\section{Nuestros profesionales}

Una eficaz suma de conocimientos: informáticos y documentalistas con experiencia

El $75 \%$ son licenciados superiores o diplomados en informática o documentación. 


\begin{tabular}{|c|c|c|c|c|}
\hline Universidad & $\mathbf{2 0 0 2}$ & $\mathbf{2 0 0 3}$ & $\mathbf{2 0 0 4}$ & $\mathbf{2 0 0 5}$ \\
\hline Burgos & 230 & 355 & 396 & 443 \\
\hline La Coruña & & & & 458 \\
\hline La Rioja & & & 272 & 658 \\
\hline León & & 640 & 368 & 567 \\
\hline Oviedo & & & 2.409 & 1.646 \\
\hline Valladolid & 1.046 & 1.153 & 1.731 & 1.000 \\
\hline Vigo & & 641 & 559 & 950 \\
\hline
\end{tabular}

Tabla 2. Descargas totales por año

\section{Resultados}

1. Datos de descargas globales de artículos desglosados por institución/año

Con las acotaciones metodológicas señaladas, la tabla 2 y la figura 1 ofrecen los datos de descargas de artículos efectuados en cada una de las instituciones académicas.

Las cifras globales de descargas no son excesivamente alentadoras, excepción hecha de las aportadas por las dos instituciones de mayor dimensión, Oviedo y Valladolid. En las universidades pequeñas y medianas la tendencia en el uso parece apuntar hacia un incremento moderado; en el caso de Burgos y León se ha podido alcanzar cierto nivel de estabilidad, teniendo en cuenta Vigo.

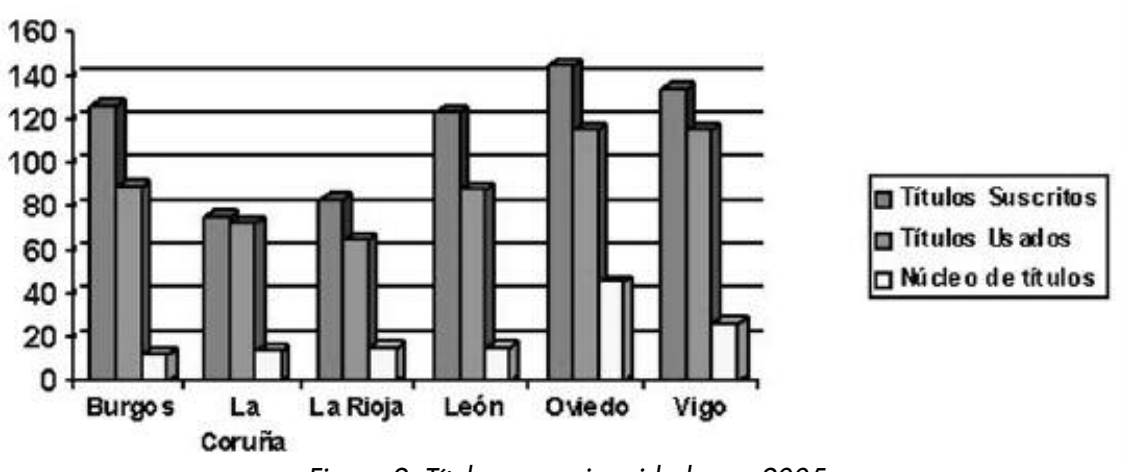

Figura 2. Títulos por universidades en 2005

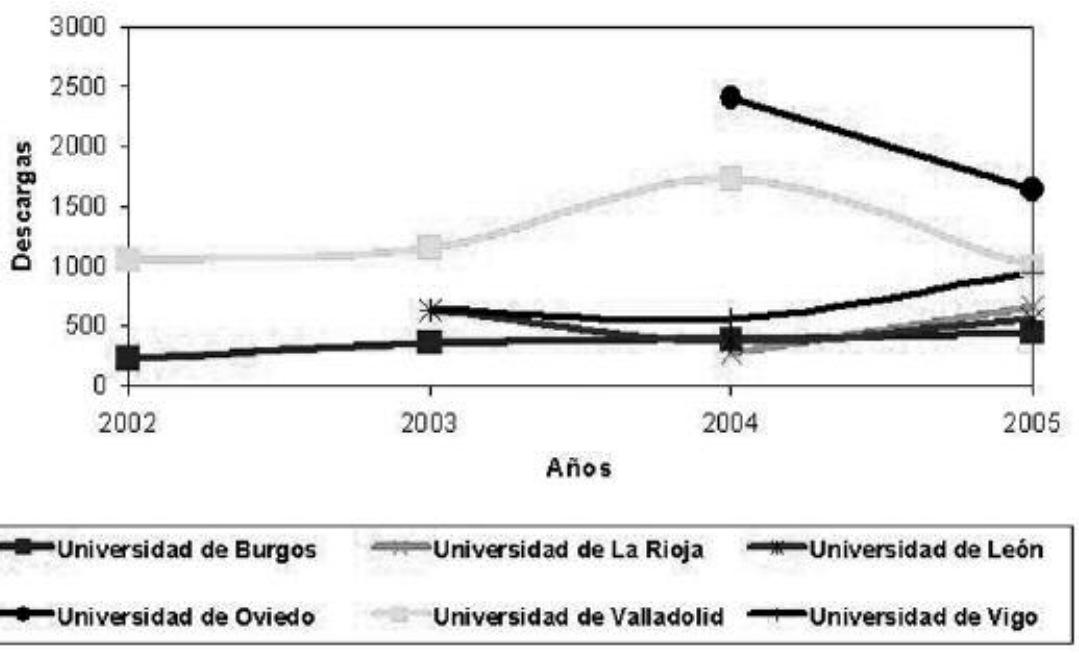

Figura 1. Evolución de las descargas totales por años que han dispuesto de los contenidos de Emerald durante un período más amplio. Digno de consideración resulta el aumento en La Rioja y en Vigo.

\begin{tabular}{|l|c|c|c|c|c|c|}
\hline & Burgos & La Coruña & La Rioja & León & Oviedo & Vigo \\
\hline Descargas artículos & 443 & 458 & 658 & 567 & 1.646 & 950 \\
\hline Títulos suscritos & 126 & 76 & 83 & 124 & 145 & 134 \\
\hline Títulos usados & 89 & 73 & 65 & 87 & 116 & 116 \\
\hline Núcleo de títulos & 12 & 13 & 15 & 15 & 45 & 26 \\
\hline
\end{tabular}

Tabla 3. Datos de Emerald en 2005
Por el contrario, las dos más grandes, Oviedo y Valladolid, con unas cifras de utilización muy considerables en 2004, sobre todo en el caso de la primera, parecen tender a una estabilización en el número de descargas a la baja.

2. Datos de descargas de cada título de revista desglosado por institución en 2005.

El umbral considerado para el establecimiento del núcleo ha tenido en cuenta el cómputo de aquellos títulos que en 2005 se habían descargado diez o más veces (tabla 3 y figura 2). 
De la relación entre títulos usados y títulos suscritos se halla la tasa de dispersión del distribuidor. De la relación entre el núcleo de títulos y títulos usados se halla la tasa de concentración de uso.

Tratándose de un suministrador pequeño, resulta comprensible que una amplia mayoría de títulos sean objeto de descarga, si bien sólo una mínima parte de los mismos reciben un uso intenso. Resultan significativas las cifras de dispersión y de intensidad de uso de La universidad de La Rioja, dado que se trata de la de menor tamaño entre las analizadas (figura 3 ).

Como se ha mencionado, la dispersión en el uso es considerable, alcanzándose en todos las instituciones el 70\%. Cabe señalar el caso de las universidades gallegas que son las que más títulos usan, alcanzando La Coruña casi el 100\%, circunstancia que puede guardar relación con ser la institución que cuenta con un menor número de revistas contratadas de Emerald. Por el contrario, las descargas efectuadas en las universidades castellano-leonesas se circunscriben a un grupo menor de publicaciones.

La intensidad de uso es escasa en todas las universidades. En ningún caso el núcleo de títulos que han recibido más de 10 descargas alcanza el 50\% (figura 4). Sólo la universidad de Oviedo supera el $30 \%$. En las universidades restantes el núcleo se encuentra entre un 13 y un $23 \%$, siendo la Burgos la que realiza un uso menos intensivo del paquete de Emerald, con un núcleo de 12 títulos, cifra próxima a las halladas en La Coruña, con 13 revistas, o La Rioja y León con 15 publicaciones. Los títulos de uso más intenso en Vigo son 26, contando Oviedo con un núcleo configurado por 45 títulos.

Se observa un patrón de comportamiento semejante en las universidades de La Coruña, La Rioja,
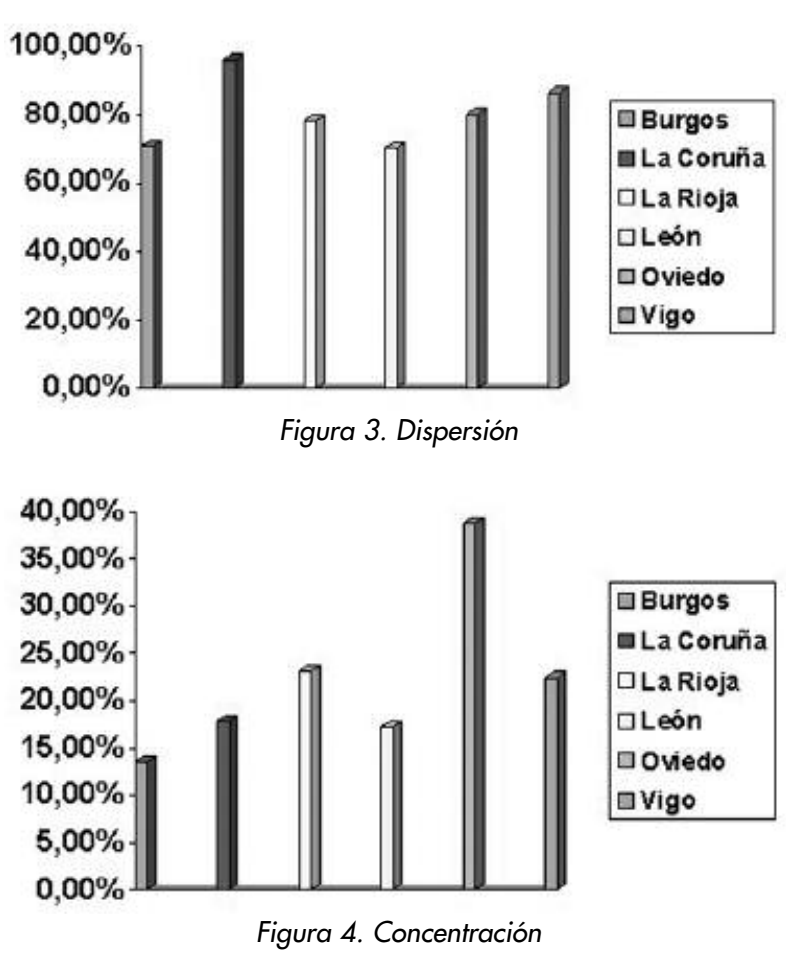

León y Vigo, en las que de un escaso $20 \%$ de los títulos ofertados se descargan casi el $70 \%$ de los artículos. En cuanto a Burgos, de un núcleo inferior al $10 \%$ de los títulos se realiza casi el 50\% de las descargas. Por lo que se refiere a Oviedo, de un núcleo ligeramente superior al $30 \%$ de los títulos se descargan el $85 \%$ de los artículos.

\section{Análisis de los títulos más empleados en el conjunto de las instituciones en 2005.}

La tabla 4 muestra los títulos de Emerald con mayor número de descargas en el conjunto de las universidades analizadas en el año 2005. Para su cómputo se han sumado las realizadas de los títulos que conforman el núcleo en las seis universidades estudiadas y se ha fijado el mínimo en 50 descargas colectivas. Se presenta, igualmente, el Factor de Impacto (FI) de 2004 según el ISI.

Los títulos que reciben un uso más intensivo se corresponden con publicaciones de marketing y de gestión. Además, figuran tres revistas del área de ingeniería: Compel, Industrial management \& data systems y Rapid prototyping journal.
El campo de la información y documentación está representado por la prestigiosa Journal of documentation, presente en el núcleo de las universidades de La Coruña, León y Vigo, en las dos primeras instituciones tal circunstancia guarda relación con el hecho de que en ambas se impartan titulaciones de documentación. Se trata de un título con el que algunas universidades contaban previamente en formato papel (Rodríguez; Alvite, 2006a).

Los títulos que ocupan las dos primeras posiciones, European journal of marketing e International journal of operations \& production management, forman parte $\mathrm{y}$, en ese mismo orden, de los seis Títulos clave que figuran en la web de la empresa para publicitar la colección.

La consolidación del uso de los títulos recogidos en la tabla 4 se verifica atendiendo a los datos tomados de años anteriores, así en 2004 European journal of marketing se encontraba en el núcleo de publicaciones de las universidades de Burgos, León, Oviedo y Vigo. Por su parte, International journal of operations \& production management figuraba en La Coruña, Oviedo y 
Vigo, Compel en La Rioja, Oviedo y Vigo mientras que Journal of documentation lo estaba tanto en $\mathrm{LaCO}$ ruña como en León.

Ninguno de los títulos listados se halla en el núcleo de las seis universidades estudiadas; de esta circunstancia se deriva que su uso varía en relación con las líneas de investigación prioritarias de las diferentes universidades. Sólo la revista International journal of service industry management está presente en el núcleo de todas las universidades, salvo en la de La Coruña. Otras tres publicaciones se hallan en el núcleo de cuatro de las universidades, entre ellos los títulos que ocupan la primera y la tercera posición en la tabla 4 , que cuentan con un uso intensivo en las universidades de Burgos, León, Oviedo y Vigo. El tercer caso se corresponde con la revista International journal of quality \& reliability management, presente en el núcleo de todas las universidades a excepción de las dos más pequeñas: La Rioja y Burgos. Las revistas restantes se hallan en el núcleo de dos o tres universidades. Por último, conviene precisar que tres de los títulos que conforman la selección realizada tienen presencia en el núcleo de una sola institución: The journal of business and industrial marketing (cuyas 107 descargas provienen únicamente de Oviedo), Managerial finance (León) y Quality assurance in education (La Coruña).

La empresa señala que 36 de los títulos distribuidos en su paquete figuran en el ISI Citation Index. Esta referencia explica que solamente 6 de los 20 títulos que configuran el núcleo global de publicaciones de Emerald hayan sido localizados en los datos que ofrece el Journal Citation Report (JCR) en 2004. Tres de los casos se corresponden con las revistas señaladas del área de ingeniería y localizadas, por tanto, en Science Citation Index. El título con mayor factor de

\begin{tabular}{|c|c|c|}
\hline Título & Total & FI \\
\hline European journal of marketing & 308 & ---- \\
\hline $\begin{array}{l}\text { International journal of } \\
\text { operations \& production } \\
\text { management }\end{array}$ & 245 & 0,556 \\
\hline Journal of services marketing & 212 & ---- \\
\hline $\begin{array}{l}\text { The learning organization: an } \\
\text { international journal }\end{array}$ & 160 & ---- \\
\hline $\begin{array}{l}\text { Journal of knowledge } \\
\text { management }\end{array}$ & 121 & ---- \\
\hline $\begin{array}{l}\text { The journal of business and } \\
\text { industrial marketing }\end{array}$ & 107 & ---- \\
\hline $\begin{array}{l}\text { International journal of quality \& } \\
\text { reliability management }\end{array}$ & 104 & ---- \\
\hline $\begin{array}{l}\text { International journal of service } \\
\text { industry management }\end{array}$ & 96 & 0,333 \\
\hline $\begin{array}{l}\text { Compel: Intl. journal for } \\
\text { computation and maths. in } \\
\text { electrical and electronic eng. }\end{array}$ & 89 & 0,180 \\
\hline $\begin{array}{l}\text { Accounting, auditing \& } \\
\text { accountability journal }\end{array}$ & 88 & ---- \\
\hline $\begin{array}{l}\text { Industrial management \& data } \\
\text { systems }\end{array}$ & 86 & 1,504 \\
\hline Journal of intellectual capital & 75 & --- \\
\hline $\begin{array}{l}\text { Supply chain management: an } \\
\text { international journal }\end{array}$ & 70 & ---- \\
\hline Managerial finance & 62 & ---- \\
\hline $\begin{array}{l}\text { Business process management } \\
\text { journal }\end{array}$ & 61 & ---- \\
\hline Journal of documentation & 61 & 1,542 \\
\hline Rapid prototyping journal & 61 & 0,585 \\
\hline $\begin{array}{l}\text { The international journal of bank } \\
\text { marketing }\end{array}$ & 61 & ---- \\
\hline Journal of consumer marketing & 58 & ---- \\
\hline $\begin{array}{l}\text { Journal of European industrial } \\
\text { training }\end{array}$ & 50 & ---- \\
\hline
\end{tabular}

Tabla 4. Revistas más usadas

impacto es Journal of documentation, único título del área de documentación ofertado por Emerald con factor de impacto.

Entre los títulos con más de 100 descargas en el cómputo global sólo International journal of operations \& production management tiene presencia en el Journal Citation Report. Se manifiesta que la mitad de los títulos con Factor de Impacto pertenecen al área de las ciencias técnicas. Podría aducirse la menor presencia de las ciencias sociales en la publicación del ISI para explicar, en gran medida, la ausencia de los títulos aquí recogidos.

\section{Discusión}

A pesar de la dificultad que supone para el análisis comparativo tanto la disparidad en el número de revistas electrónicas suscritas por las distintas instituciones estudiadas como la diversidad en el tamaño de las mismas, parece apreciarse cierta consolidación en el uso de Emerald que podría obedecer a la contratación paralela de diversos proveedores de recursos electrónicos. Todas las universidades estudiadas mantienen suscritos los paquetes multidisciplinares de ScienceDirect, Springer-Kluwer y Wiley. La Universidad de Oviedo carece de los dos últimos y de ahí quizá el uso intensivo que esta institución realiza de Emerald.

Consideramos, no obstante, que en las universidades objeto de estudio la extensión en el uso es todavía susceptible de incrementarse incidiendo las bibliotecas en la forma- 
Transmitir el conocimiento y los instrumentos característicos de la inteligencia competitiva (IC) que permiten al participante establecer un sistema formal de recogida, selección, análisis y distribución de la información sobre el entorno y los competidores de la empresa y gestionar y administrar el departamento o la unidad de inteligencia.

A quién se dirige:

- Responsables de investigación de mercado

- Responsables de marketing

- Personal de apoyo a la planificación estratégica de la empresa

- Responsables de información científica y técnica

- Responsables de sistemas de información (webmasters)

- Responsables de la gestión del conocimiento

- Documentalistas corporativos

- Directivos de empresa que desean conocer mejor el potencial de la inteligencia competitiva

\section{Calendario:}

- 4a edición: del 1 de noviembre de 2006 al 31 de enero de 2007.

- $5^{a}$ edición: del 16 de abril al 13 de julio de 2007.

Web:

www.idec.upf.edu/cicol

Contacta con nosotros y te atenderemos de manera personalizada

Tel. +34 935421850 www.idec.upf.edu info@idec.upf.edu Balmes, 132 ción de usuarios y en la personalización en la organización de colecciones. Como constatan Borrego et al. (2005) en el informe elaborado con datos de una encuesta realizada por los usuarios de las bibliotecas que conforman el $C B U C$, no existe resistencia al cambio del formato papel al electrónico, sino más bien desconocimiento y limitación en las habilidades tecnológicas entre el personal docente e investigador que realiza un menor uso de los recursos digitales.

Creemos, asimismo, que el uso de todos los proveedores podrá verse potenciado gracias a la facilidad reciente que posibilita el acceso a los contenidos electrónicos desde el exterior de la institución, ahora bien, el paquete objeto de análisis se destina mayoritariamente a los científicos de ciencias sociales, no adscritos a las áreas que hacen un uso prioritario de las publicaciones periódicas, de ahí que consideremos que el incremento de uso será moderado.

\section{"Tratándose de un suministrador pequeño, resulta comprensible que una amplia mayoría de títulos sean objeto de descarga, si bien sólo una mínima parte de los mismos recibe un uso intenso"}

No se ha apreciado en el uso de este distribuidor una correspondencia proporcional entre el consumo de información electrónica y el tamaño de la institución. De hecho, las universidades de dimensiones más reducidas en algunos casos se aproximan o superan el uso realizado por otras de tamaño medio, sien- do significativo el caso de La Rioja con cifras elevadas de descarga.

Aunque no presentamos datos singularizados por meses, se ha observado que durante los primeros momentos de funcionamiento de los paquetes se producen dos fenómenos que dificultan la valoración de la evolución de uso, el curioseo de los investigadores y las pruebas de los bibliotecarios. Así mismo, existen incrementos aislados en el uso de diferentes títulos que pueden obedecer a la realización de trabajos puntuales por parte del alumnado.

El trabajo de Urbano et al. (2004) que analiza el uso de varios proveedores de contenidos en las universidades catalanas constata una importante dispersión en Emerald (en torno a un 45\%), corroborado igualmente por la empresa (Evans; Peter, 2005), y que se confirma al alza en las universidades estudiadas en 2005 , donde la cifra alcanza el 
$70 \%$. Asimismo, el estudio catalán señala la limitada intensidad de uso de Emerald, extremo ratificado en el presente trabajo donde ninguna universidad alcanza en su núcleo un $50 \%$ de los títulos, sólo la Universidad de Oviedo supera el 30\% de revistas usadas intensivamente.

Se aprecia, igualmente, que muchos títulos reciben un uso meramente circunstancial, lo que nos conduce a considerar que a la larga las bibliotecas realizarán una selección de la información que necesitan y el modelo Big Deal evolucionará. No obstante, dicha selección habrá de realizarse a partir del uso real de los recursos y no a priori, condicionada por factores económicos como se venía produciendo. En el caso de Emerald cabe señalar que se realiza un uso considerablemente extensivo, que puede obedecer a las reducidas dimensiones del paquete, de ahí que los datos globales presentados resulten más positivos que los obtenidos por Sanville, quien refiriéndose a la utilización de varios paquetes en las universidades de Ohio, señalaba que del $45 \%$ de los títulos ofertados se descargaban únicamente entre un $8 \mathrm{y}$ un $10 \%$ de los artículos.

En el caso de las universidades analizadas, del $80 \%$ de los títulos disponibles se descargan sólo el $30 \%$ de los artículos. Únicamente Burgos y Oviedo se alejan del patrón común y en sentidos inversos. La primera, con un núcleo de títulos muy reducido, realiza un uso muy disperso, mientras que Oviedo concentra, contrariamente, el uso que realiza de los contenidos suministrados en un amplio núcleo de títulos.

Del núcleo de títulos más usados no puede inferirse que se realice un uso más intensivo de títulos tradicionalmente reputados con Factor de Impacto, aspecto que podría vincularse a la idiosincrasia de los títulos distribuidos, mayoritariamente de ciencias sociales.
El presente análisis ha pretendido ofrecer información útil sobre el uso de revistas electrónicas en varias universidades en el momento de la transición en España del formato papel al formato electrónico. Proporciona datos objetivos imprescindibles para el desarrollo de las colecciones con la intención de que su estudio y análisis permitan a las bibliotecas el mantenimiento del liderazgo en la provisión de información científica.

\section{Nota}

1. Guía universidades: catálogo oficial de titulaciones 2005-2006, curso 05-06. CRUE, Ministerio de Educación y Ciencia, Universia, 2005.

http://www.mec.es/educa/ccuniv/html/guiacrue/ CD-guiacrue/index.htm

Estadística universitaria: curso 2004-2005. Consejo de Coordinación universitaria, 2005

\section{Bibliografía}

Alvite Díez, M. L.; Rodríguez Bravo, B. "Distribuidores de contenidos electrónicos: acceso, interfaz y funcionalidades". En: 9as. Jornadas españolas de documentación, 2005, pp. 29-46.

Anglada, L.; Comellas, N.; Roig, J.; Ros, R.; Tort, M. "Licensing, organizing and accessing e-journals in the Catalan university libraries". En: Serials, 2003, v. 16, n. 3, pp. 299-306.

Borrego, A. (dir). Estudi d'usuaris de les revistes electròniques del CBUC. Consorci de Biblioteques Universitàries de Catalunya, 2005. Consultado en: 04-05-06.

http://www.recercat.net/bitstream/2072/1439/1/E STUDI+USUARIS.pdf

Davis, P. M.; Price, J. S. “eJournal interface can influence usage statistics: implications for libraries, publishers, and project Counter". En: Journal of the American Society for Information Science and Technology, 2006, v. 57, n. 9, pp. 1.243-1.248.

Eason, K.; Richardson, S.; Yu, L. "Patterns of use of electronic journals". En: Journal of documentation, 2000, v. 56, n. 5, pp. 477-504.

Evans, P.; Peter, J. Analysis of the dispersal of use for journals in Emerald Management Xtra $(E M X)$. Emerald Group Publishing Limited, 2005 .

Frazier, K. "The librarians' dilemma: contemplating the costs of the big deal". En: D-lib magazine, 2001a, v. 7, n. 3. Consultado en: 02-05-06.

http://www.dlib.org/dlib/march01/frazier/03frazi er.html

Frazier, K. "To the editor: letters in response to the opinion piece, The librarians' dilemma: contemplating the costs of the big deal". En: $D-l i b$ magazine, 2001b, v. 7, n. 4. Consultado en: 02-05-06. http://www.dlib.org/dlib/april01/04letters.html

Ingenta Institute. The consortium site licence: is it a sustainable model? Edited proceedings of a meeting held on 24th September 2002, London, organised by the Ingenta Institute. Oxford: Ingenta Institute, 2002.

Luther, J. "White paper on electronic journal usage statistics". $2^{\text {nd }}$ ed., Washington D. C. Council on Library and Information Resources, 2001. Consultado en: 04-07-06.

http://www.clir.org/pubs/reports/pub94/pub94.pdf

Peters, T. A. "What's the use? The value of e-resource usage statistics". En: New library world, 2002, v. 103, n. 1.172/1.173, pp. 39-47.

Rodríguez Bravo, B.; Alvite Díez, M. L.

"Análisis de los contenidos distribuidos por proveedores de publicaciones electrónicas". En: El profesional de la información, 2004, v. 13, n. 6 , pp. 441-449.

Rodríguez Bravo, B.; Alvite Díez, M. L. "Survey of the providers of electronic publications holding contracts with Spanish university libraries". En D-lib magazine, 2005, April, v. 11, n. 4.

http://www.dlib.org/dlib/april05/alvite/ 04alvite.html

Rodríguez Bravo, B.; Alvite Díez, M. L. "The use of electronic journals in academic libraries in Castilla and León (Spain)". En: "Libraries without walls 6: evaluating the distributed delivery of library services". London: Facet Publishing, 2006a, pp. 125-137.

Rodríguez Bravo, B.; Alvite Díez, M. L. "The use of electronic journals in academic libraries in the Northwest of Spain". En: LIDA: libraries in the digital age 2006, 2006b.

Rowse, M. "The consortium site license: a sustainable model?" En: Libri, 2003, v. 53, n. 1, pp. $1-10$.

Rush-Feja, D.; Siebeky, U. "Evaluation of usage and acceptance of electronic journals". En: D-lib magazine, 1999, October, v. 5, n. 10. Consultado en: 02-05-06. http://www.dlib.org/dlib/october99/rusch-feja/ 10rusch-feja-summary.html

Sanville, T. "A method out of the madness: OhioLink's collaborative response to the serial crisis: four years later progress report". En: $\mathrm{Se}$ rials, 2001, v. 14, n. 2, pp. 163-177.

Tenopir, C. Use and users of electronic library resources: an overview and analysis of recent research studies. Washington, D. C.: Council on Library and Information Resources, 2003. Consultado en: 22-04-06.

http://www.clir.org/pubs/reports/pub120/ pub120.pdf

Urbano, C. (et al.). The use of consortially purchased electronic journals by the CBUC (2000-2003). En: D-lib magazine, 2004, v. 10, n. 6. Consultado en: 02-05-06. http://www.dlib.org/dlib/june04/anglada/ 06anglada.html

Blanca Rodríguez, Bravo y $M^{a}$ Luisa Alvite Díez, Área de Biblioteconomía y Documentación, Universidad de León. dphbrb@unileon.es luisa.alvite@unileon.es 